Thomas Hapke

\title{
Informationskompetenz 2.0 und das Verschwinden des „Nutzers“*
}

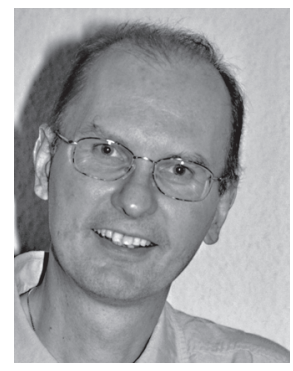

Ausgehend von einer sich wandelnden Rolle des Nutzers von Informationssystemen im Web 2.0 wird das Konzept Informationskompetenz kritisch hinterfragt. Fünf Thesen zur Informationskompetenz 2.0, verbunden mit Anregungen auf weiterführende Literatur, schlagen einen Perspektivwechsel der in deutschen Bibliotheken vorherrschenden Sicht auf Informationskompetenz hin zu einem ganzheitlicheren Verständnis von Informations- und Lernprozessen vor.

Information literacy 2.0 and the disappearance of the user

Starting from the changing role of the user in information systems in the Web 2.0 this paper critically questions the concept of information literacy. Five theses on information literacy 2.0 as well as suggestions for further reading offer the possibility to change the perspective of the view on information literacy predominating in German libraries in the direction of a more holistic view of information and learning processes.

Maîtrise de l'information 2.0 et la disparition de l'usager

En partant du rôle se modifiant de l'usager des systèmes d'information dans le Réseau 2.0, le projet de la maîtrise de l'information est remis en question critiquement. Liée avec des suggestions sur la littérature, cinq thèses sur la maîtrise de l'information proposent une perspective alternative de la vue sur la maîtrise de l'information prédominante aux bibliothèques allemandes, une perspective à une compréhension des procédés d'information et d'apprentissage plus intégrante.

\section{Der Nutzer in einer sich ständig verändernden} Welt der Information

Das Thema Förderung von Informationskompetenz als Aufgabenfeld von Bibliotheken hat sich auch in Deutschland fest etabliert. Davon zeugen häufig erscheinende Aufsätze zum Thema ${ }^{1}$ sowie regelmäßig stattfindende Vorträge auf Konferenzen. Aber die Welt der Information verändert sich rasant weiter und das Aufkommen des so genannten Web 2.0 bringt auch für Bibliotheken weitere neue Herausforderungen ${ }^{2}$. Was bedeutet nun das Web 2.0 für die Förderung von Informationskompetenz durch Bibliotheken in der Zukunft? Wie verändert sich das, was als Informationskompetenz aufgefasst wird in einer sich ständig ändernden elektronischen Umwelt? Welche Auswirkungen hat das Web 2.0. auf das Informationsverhalten der Nutzenden? Wie können Komponenten des Web 2.0 zur Förderung von zeitgemäßer Informationskompetenz genutzt werden? Ausgehend von der sich verändernden Rolle des Nutzers im Web 2.0 werden in diesem Aufsatz fünf Thesen als Ausgangspunkt für eine sich verändernde Sicht auf Informationskompetenz vorgeschlagen und durch Anregungen für weiterführende Lektüre ergänzt. Informationssysteme beobachten und erfassen heute vielfältige Nutzeraktivitäten und lernen somit von den Nutzenden. Gleichzeitig werden Informationssysteme auch zu Lern- und Kommunikationssystemen, wenn man die sich immer weiter verbreitenden Lernmanagementsysteme des E-Learnings betrachtet. Hier lernt dann der Nutzer vom und mit dem System. Aber mehr noch, der "Tod des Nutzers" - so einige amerikanische Autoren ${ }^{3}$ in Anlehnung an Roland Barthes - ist absehbar: Der Nutzer im Zeitalter des Web 2.0 wird immer mehr zum Mit-Produzenten eines Systems, das Gleichgesinnte in Denk-, Lern- und Praxisgemeinschaften vereinigt. Der Nutzer wird damit Teil von Informationssystemen, die sich immer mehr zu Kommunikationssystemen entwickeln.

Dieser Artikel ist lizensiert unter der Creative Commons Attribution 2.0 Germany License < http://creativecommons. org/licenses/by/2.0/de/>.

1 Siehe einen aktuellen Überblick bei Hütte, Mario: Zur Vermittlung von Informationskompetenz an Hochschulbibliotheken - Entwicklung, Status quo und Perspektiven. In: Bibliothek 30 (2006) S. 137-167.

2 Im Aufsatz von Danowski, Patrick und Lambert Heller: Bibliothek 2.0 - Die Zukunft der Bibliothek? In: Bibliotheksdienst 40 (2006) S. 1 259-1 271 (Preprint <http://eprints.rclis.org/ archive/00007618/>, besucht am 2.4.2007) wird hinsichtlich der Zukunft der in Bibliotheken arbeitenden Menschen und deren zukünftigen Funktionen implizit auch der Bereich Informationskompetenz gestreift!

3 Rosenbaum, Howard; Davenport, Elisabeth; Lievrouw, Leah A. und Ronald E. Day: The death of the user. In: 2003 Annual Meeting - Humanizing Information Technology: From Ideas to Bits and Back - Proceedings of the American Society for Information Science and Technology 40 (2003) S. $429-430$. 


\subsection{Die klassische Sicht auf Nutzer und Informa- tionssystem}

Das Verhältnis des Nutzers zu den von inm genutzten Informationssystemen hat sich in den letzten Jahren gewandelt, seien diese Informationssysteme Datenbanken, andere Angebote im Netz oder auch eine Institution wie eine Bibliothek. Im klassischen Information-Retrieval-Modell standen sich Nutzer und System noch auf zwei Seiten wie Subjekt und Objekt gegenüber: Der Recherchierende drückte seinen Informationsbedarf in seiner Anfrage mit Hilfe von Suchbegriffen aus, die dann in Form eines Abgleichs (Matching) mit den in der Datenbank enthaltenen Begriffen aus den Beschreibungen der Texte (Metadaten) oder den Texten selbst das Suchergebnis produzierten. Dabei konnten die Produzenten des Informationssystems die Metadaten oder Volltexte inrerseits noch mit zusätzlichem Vokabular anreichern. Nutzer und Informationssystem interagierten aber weitgehend nur über die Suchanfrage und das Rechercheergebnis. Der Nutzer als selbstbestimmte und autonome Person mit bewusstem oder auch unbewusstem Informationsbedürfnis und individuellen Zielen be-,nutzt" das Informationssystem, um sein Ziele zu erreichen. Das Informationssystem selbst wird durch die Recherchen des Nutzers nicht beeinflusst. Oft tritt zwischen Nutzer und System ein Vermittler, z. B. eine Person, eine Oberfläche oder eine andere Form von personalem oder maschinellem Informationsassistenten ${ }^{4}$.

Die klassische Sicht auf den Nutzer in Bibliotheken ist dabei die Sicht des Experten und Informationsspezialisten. Der Nutzer als ",monologisches Subjekt" ${ }^{\text {5 }}$ mit seinen physischen, kognitiven und affektiven Eigenschaften ist unabhängig von anderen Individuen um ihn herum. Der Informationsspezialist steht aus dieser Sicht in einem Verhältnis zum Nutzer, die dem Verhältnis von Arzt/Patient bzw. Erwachsener/Kind nahe kommt: der sichere, rationale, neutrale, bewusste, reflektierende Experte und der oft unsichere, frustrierte, ängstliche und desorientierte Nutzer.

\subsection{Wandlung des Verhältnisses zwischen Infor- mationssystem und Nutzer}

Die aktuellen Entwicklungen im Internet verändern heute das Verhältnis von Nutzer und Informationssystem immer mehr. Nutzer und System verhalten sich nicht mehr nur wie Subjekt und Objekt, wobei das Subjekt das Objekt nutzt, ohne dieses zu verändern oder ohne dass es von diesem sichtbar verändert wird. Viel stärker als früher beeinflusst, verändert und erweitert der Nutzer heute das benutzte Recherchesystem. Nicht nur durch Nutzermodellierung bzw. Adaptierung dessen Verhaltens rücken die Sphären des Nutzers und des jeweiligen Informationssystems enger zusammen. Nutzer und Informationssystem agieren als Lernende.

Einerseits gibt es heute Informationssysteme, die vom Nutzer lernen. Systeme wie zum Beispiel BibTutor lernen vom Nutzer, indem der persönliche Informationsbedarf durch Nutzereingabe abgebildet wird, und indem die Rechercheschritte und vom Nutzer abgerufenen SupportKomponenten erfasst und ausgewertet werden ${ }^{6}$. Das System lernt aus den Nutzereingaben, der Nutzer wiederum lernt durch spezifische Rückmeldungen des Systems.
So kann die individuelle Nutzung eines Informationssystems sogar individuelle Recherchen anderer Nutzer beeinflussen. Zum Beispiel ist in einem Recherchesystem wie Amazon ein "Recommender-System“ integriert, das die konkrete Suche mit Empfehlungen aus RechercheErgebnissen bzw. Nutzungsdaten anderer Nutzer begleitet. Andererseits stellen die inzwischen weit verbreiteten Lernmanagementsysteme des E-Learning nichts anderes als digitale Bibliotheken von Lernobjekten dar, mit denen der Nutzer selbst etwas lernen kann.

\subsection{Das Verschwinden des Nutzers}

Heute wird der Nutzer verstärkt als Kunde, Verbraucher oder Konsument gesehen. Informationskompetenz ist dann z. B. die Fähigkeit, aufgeklärte und informierte, d. h. bewusste Konsumentscheidungen zu treffen ${ }^{7}$. Das Informationsverhalten in Alltag und Freizeit prägt auch die Kunden von professionellen Informationssystemen. In einer von „Amazoogle“8 geprägten Welt sind Konsumenten heute nicht mehr nur passiv, sondern aktiv. Der Informationssuchende agiert als Spieler ${ }^{9}$ und Lernender ${ }^{10}$.

Die Nutzung von Informationssystemen, die mit Komponenten des Web 2.0, mit sozialer Software wie Weblogs und Wikis, realisiert werden, umfasst normalerweise gleichzeitig die Möglichkeit, selbst zu agieren, sein eigenes Wissen und seine eigenen Fähigkeiten anderen zur Verfügung zu stellen bzw. diese mit anderen zu teilen. Damit wird der Nutzer selbst Teil des Informationssystems, tritt als Informationsvermittler auf bzw. wandelt sich zum Mitproduzenten des Systems. Der konkrete Nutzer selbst ist nicht mehr nur als ein autonomes Individuum sichtbar, sondern Teil einer Gemeinschaft, in der er agiert, oder in

4 Kuhlen, Rainer: Die Konsequenzen von Informationsassistenten: was bedeutet informationelle Autonomie oder wie kann Vertrauen in elektronische Dienste in offenen Informationsmärkten gesichert werden? Frankfurt a. M. 1999.

5 Zum Folgenden siehe Tuominen, Kimmo: User-centred discourse: an analysis of the subject positions of the user and the librarian. In: The Library Quarterly 67 (1997) S. 350-371.

6 Hapke, Thomas; Memmel, Martin und Sandra Zilles: Benutzerzentrierte Modellierung des Rechercheprozesses in BibTutor. In: Content: 28. Online-Tagung der DGI, 58. Jahrestagung der DGI, Proceedings hrsg. v. Marlies Ockenfeld. Frankfurt a. M.: Deutsche Gesellschaft für Informationswissenschaft und -praxis, 2006. Hier: S. 123-133. Siehe auch die Website des Projektes unter <http://www.bibtutor.de>, besucht am 2.4.2007.

7 Pawley, Christine: Information literacy: a contradictory coupling. In: The Library Quarterly 73 (2003) S. 422-452.

8 Dempsey, Lorcan: The (Digital) Library Environment: Ten Years After. In: Ariadne, Issue 46, February 2006, <http://www. ariadne.ac.uk/issue46/dempsey/>, besucht am 2.4.2007.

9 Nicholas, David und Tom, Dobrowolski: The ,information player': a new and timely term for the digital information user. In: Handbook of information management. Ed. by Alison Scammell, Association for Information Management. 8. Ed. London 2001. Hier S. 513-522.

10 Hepworth, Mark: Information literacy from the learners perspective. In: Information and IT literacy: enabling learning in the 21st century. Ed. by Allan Martin and Hannelore Rader. London 2003. Hier S. 217-233. 
die er integriert ist. Gerade in Untersuchungen zur Nutzung digitaler Bibliotheken ist dies immer mehr Gegenstand der Forschung ${ }^{11}$. Der Nutzer ist als „organizational individual"12 eingebunden in ein sozio-technologisches Netzwerk mit kulturellen, politischen und wirtschaftlichen Elementen. Er trägt als „,sozialer Akteur“13 mit zur Konstitution und zum Design von Informationssystemen der Zukunft bei. So gehört auch das so genannte „Tagging“, die gemeinschaftliche Erschließung von Webseiten, Büchern u.a., zu diesen Phänomenen, in denen die Nutzer aktiv in Informationssystemen wie del.icio.us, LibraryThing oder Flickr die Inhalte mit selbst gewählten Stichwörtern versehen ${ }^{14}$.

Die Rollen zwischen Schreibendem und Lesendem, zwischen dem Produzenten und dem Konsumenten von Information gleichen sich immer mehr an. So wie Walter Benjamin schon 1934 die Wandlung des Lesers zum Autor beschrieben hat ${ }^{15}$, - eine Wandlung, die durch die vielfältigen Möglichkeiten des Internets in den letzten Jahren fast schon Alltag geworden ist - so wird der Nutzer eines Informationssystems immer mehr zu einem Akteur innerhalb des Systems und macht sein Wissen und seine Fähigkeiten öffentlich, d. h. er publiziert und kommuniziert.

\section{Informationskompetenz 2.0}

Macht es überhaupt Sinn, so etwas wie Informationskompetenz 2.0 beschreiben zu wollen? Gibt es Informationskompetenz 2.0 überhaupt? Die Bezeichnung ist zuerst von Edlef Stabenau eingeführt worden. Sie stand als eher ironisch gemeinte Überschrift im Weblog Netbib über einigen Anmerkungen zum Design der Website <informationskompetenz.de $>^{16}$. Die folgenden Thesen dieses Aufsatzes zu Informationskompetenz 2.0 charakterisieren fünf durchaus aufeinander bezogene Komponenten eines modernen Verständnisses von Informationskompetenz im Zeitalter des Web 2.0. Sie beziehen sich auf eine besonders in der internationalen Literatur anzutreffende kritisch-konstruktive Auffassung zur Informationskompetenz, die angesichts der Verbreitung sozialer Software verstärkt Beachtung finden sollte. Innerhalb der bibliothekarischen Diskussion sind besonders in Deutschland mehr kritische Stimmen nötig, die ja häufig auch neue Einsichten und Ideen geben $^{17}$. Die Thesen dieses Aufsatzes (vgl. Tabelle 1) enthalten einen Vorschlag für einen Perspektivwechsel der in Deutschland bisher vorherrschenden Sicht auf Informationskompetenz.

1. Informationskompetenz 2.0 umfasst ein ganzheitliches Verständnis von Informations- und Lernprozessen.

2. Informationskompetenz 2.0 ist eine von vielen Schlüsselkompetenzen.

3. Informationskompetenz 2.0 ist eine ,,Lernerfahrung".

4. Informationskompetenz 2.0 ist im Web 2.0 ein Prozess, umfasst nicht nur Lernen mit und durch Information, sondern verstärkt Lernen über Information und Wissen.

5. Informationskompetenz-2.0-Förderung nutzt Hilfsmittel des Web 2.0.

Tabelle 1: Thesen zur Informationskompetenz 2.0
Thematisiert wird damit auch die Frage nach der Veränderung des Verständnisses von Informationskompetenz in einer sich ständig ändernden elektronischen Umwelt. So betraf dies in der nahen Vergangenheit z. B. die Frage, welche Auswirkungen die parallele Suche in mehreren Datenbanken, wie sie Portale bieten, auf die Kompetenzen ihrer Nutzer hat. Es war bisher für Nutzende wichtig zu wissen, welcher Unterschied zwischen einer selbständigen Veröffentlichung, also einem Buch oder einer Zeitschrift, und einer bibliographisch unselbständigen Veröffentlichung, also z. B. einem Zeitschriftenaufsatz, besteht. Je nachdem, wonach er suchte, musste der Endnutzer eine andere Datenbank auswählen: für die Recherche nach einem Buch z. B. den Bibliothekskatalog, der in der Regel keine Aufsätze enthält, für die Recherche nach einem Aufsatz eine fachspezifische Aufsatz-Datenbank. Bleibt diese Unterscheidung in Zukunft weiterhin wichtig, wenn wir einheitliche Benutzer-Oberflächen haben, in denen Aufsatzdatenbanken, Bibliothekskataloge u.a. integriert sind?

Welche Auswirkungen hat heutzutage speziell das Web 2.0. auf das Informationsverhalten der Nutzenden? Welche Form von Informationskompetenz wird in der Welt sozialer Software vom Informationssuchenden benötigt? Gibt es in Zukunft überhaupt noch so etwas wie einen „Informationssuchenden", wie wir inn uns aus bibliothekarischer Sicht vorstellen? Die im ersten Teil dieses Aufsatzes beschriebene Entwicklung lässt gerade die letzte Frage als durchaus gerechtfertigt erscheinen.

11 So werden in Bishop, Ann Peterson; Van House, Nancy A. und Barbara P. Butterfield (Eds.): Digital library use: social practice in design and evaluation. Cambridge, Mass. 2003, digitale Bibliotheken unter anderem als Ökosysteme beschrieben, und der Nutzer wird als Teil von Gemeinschaften angesehen, deren „Wissensarbeit“ gemeinsame Erkenntnisse „konstruiert“.

12 Siehe Rosenbaum et al. (Anm. 3).

${ }^{13}$ Lamb, Roberta und Rob Kling: Reconceptualizing users as social actors in information systems research. In: MIS Quarterly 27 (2003) 2, June. Online: <http://www.misq.org/ archivist/bestpaper/Lamb.pdf>, besucht am 2.4.2007.

${ }_{14}$ Macgregor, George und Emma McCulloch: Collaborative tagging as a knowledge organisation and resource discovery tool. In: Library review 55 (2006) 5, S. 291-300.

15 So schrieb Benjamin, Walter: Die Zeitung (1934). In: Benjamin, Walter: Gesammelte Schriften. 2. Band, 2. Teil. Frankfurt a. M. 1977, hier S. 628-629: „Indem nämlich das Schrifttum an Breite gewinnt, was es an Tiefe verliert, beginnt die Unterscheidung zwischen Autor und Publikum ... zu verschwinden. Der Lesende ist jederzeit bereit, ein Schreibender, nämlich ein Beschreibender oder auch ein Vorschreibender zu werden. Als Sachverständiger - und sei es auch nicht für ein Fach, vielmehr nur für den Posten, den er versieht - gewinnt er einen Zugang zur Autorenschaft..."

${ }^{16}$ Am 31.8.2006 unter <http://log.netbib.de/archives/2006/08/31/ informationskompetenz-20/>, besucht am 2.4.2007.

17 Eine in Deutschland singuläre kritische Sicht auf Informationskompetenz bietet, verbunden mit einer Auswertung internationaler Literatur: Ingold, Marianne: Das bibliothekarische Konzept der Informationskompetenz: ein Überblick. Berlin 2005. Online: <http://www.ib.hu-berlin.de/ kumlau/ handreichungen/h128/>, besucht am 2.4.2007. 


\subsection{These 1: Informationskompetenz 2.0 umfasst ein ganzheitliches Verständnis von Informa- tions- und Lernprozessen}

Über effiziente Recherche- und Navigationsstrategien hinaus umfasst Informationskompetenz vor allem die Kreativität, den eigenen Informations- und Lernprozess bewusst und bedarfsgerecht zu gestalten. Informationskompetenz 2.0 ist nicht nur ein durch Standards beschreibbares Kompetenzfeld, sondern umfasst die gesamte Vielfalt bzw. Multidimensionalität des möglichen Begriffsverständnisses von Informationskompetenz. Informationskompetenz wird nach Christine Bruce definiert als die Summe der verschiedenen Formen, in der sie erfahren wird ${ }^{18}$. Pädagogisch-didaktische Aktivitäten zu ihrer Förderung und als Unterstützung für Lernende ermöglichen es, so Ola $\mathrm{Pi}$ lerot, das Repertoire an Erfahrungen beim Suchen, Finden und Nutzen von Information auszuweiten und zu verändern. In einem Aufsatz von Bruce mit zwei australischen Kolleginnen werden sechs verschiedene Sichten auf Informationskompetenz unterschieden, eine Sicht auf den Inhalt, eine andere auf Kompetenzen, auf das „Lernen zu Lernen", auf die persönliche Relevanz, auf die soziale Bedeutung sowie eine die gerade erwähnten fünf Bedeutungsnuancen zusammen- und umfassende relationale Sicht auf Informationskompetenz ${ }^{19}$. Limberg und Sundin differenzieren eine quellen-, verhaltens-, prozess- und kommunikationsorientierte Betrachtungsweise von Informationskompetenz ${ }^{20}$. Hier erwähnt ist auch ein Ansatz, Informationskompetenz als eine Form von „sociotechnical practice" zu sehen. Gemeint ist hier eine Praxis, in der es darauf ankommt, soziale, ideologische und materiell-technische Kontexte zu kennen und zu verstehen, in denen Information, Wissen und deren Medien bzw. technischen Werkzeuge produziert bzw. genutzt werden ${ }^{21}$. Informationskompetenz ist dann eher ein durch individuelle und gemeinschaftliche sowie formelle und informelle Erfahrungen gewonnenes Verständnis für das Funktionieren von Informations-, Publikations- und Lernprozessen. In einem gerade erschienenen Buch zum Engagement von Studierenden und Informationskompetenz wird letztere als „way of thinking", als "dispositional habit", also als der Neigung entsprechenden Lebensgewohnheit, aber auch als "set of cultural practices“ gesehen ${ }^{22}$.

Diese in den obigen Ansätzen sichtbare, eher holistische Sicht auf Informationskompetenz relativiert die Rolle von Standards zur Informationskompetenz, wie z. B. die aus den übersetzten ACRL-Standards entwickelten aus Baden-Württemberg ${ }^{23}$. Standards bilden eine gute Hilfe und Basis für die eigene Reflexion und mögen aus politischen und Marketing-Gesichtspunkten wichtig erscheinen, aber Informationskompetenz ist mehr, als sich in Standards abbilden lässt. Trotzdem können Standards dazu dienen, die Reflexion darüber zu strukturieren, welche Bedeutung das Web 2.0 auf den Begriff und die Praxis von Informationskompetenz hat ${ }^{24}$.

Informationskompetenz 2.0 variiert die Sichtweisen und Perspektiven auf Informationskompetenz, Annemaree Lloyd spricht sogar von „information literacy landscapes“25.Die Sicht von Bibliotheken reicht nicht aus, um alle Facetten sichtbar zu machen. So kann z. B. eine betriebliche, unternehmensorientierte Sicht auf Informationskompetenz ${ }^{26}$ zeigen, dass im Unternehmen die Suche nach Information ganz klar weniger wichtig ist als die Informationsnutzung und -produktion, sowie weniger wichtig als das Problem der Bewältigung der Informationsflut. Auch unklare, komplexe und manchmal wenig transparente Informationsprozesse im Unternehmen und beschränkter Zugang zu Informationsquellen sind oft problematischer als die Nutzung

18 So bei Pilerot, Ola: Information literacy at a distance - collaboration between a university library and two public libraries. In: Second International Conference on Information and IT Literacy. Glasgow Caledonian University 2003. Online: <http://www.elit-conf.org/elit2003/papers/ppt/pilerot. pdf>, besucht am 2.4.2007. Hier S. 5.

${ }^{19}$ Bruce, Christine; Edwards, Sylvia und Mandy Lupton: Six frames for Information literacy education. In: ITALICS (Innovations in Teaching and Learning in Information and Computer Sciences) 5 (2006) 1. Online: <http://www.ics. heacademy.ac.uk/italics/vol5-1/pdf/sixframes_final\%20_1_ .pdf>, besucht am 2.4.2007.

${ }^{20}$ Limberg, Louise und Olaf Sundin: Teaching information seeking: relating information literacy education to theories of information behaviour. In: Information Research 12 (2006) 1, paper 280. Online: <http://InformationR.net/ir/12-1/ paper280.html>, besucht am 2.4.2007.

${ }^{21}$ Siehe S. 340 in Tuominen, Kimmo; Savolainen, Reijo und Sanna Talja: Information literacy as a sociotechnical practice. In: Library Quarterly 75 (2005) S. 329-345.

22 Student engagement and information literacy. Ed. by Craig Gibson. Chicago 2006. Hier S. VIII ff.

23 "Standards der Informationskompetenz für Studierende. Hrsg. Netzwerk Informationskompetenz Baden-Württemberg (NIK-BW)" <http://www.informationskompetenz.de/ fileadmin/DAM/documents/Standards\%20der\%20Inform_ 88.pdf>, besucht am 2.4.2007. Zur Situation der Förderung von Informationskompetenz in Baden-Württemberger Bibliotheken vergleiche Sühl-Strohmenger, Wilfried: Das Netzwerk Informationskompetenz der baden-württembergischen Hochschul- und Landesbibliotheken. In: B.I.T.online 9 (2006) S. $205-212$.

24 So nutzen Deitering, Anne-Marie und Rachel Bridgewater (Research instruction in a Web 2.0 world: an ACRL/Instruction Section Current Issue Discussion Forum. ALA Conference, New Orleans, 25 June 2006, Online: ALA | Instruction Section Current Issues Discussion Forum - Annual 2006 <http://www.ala.org/ala/acrlbucket/is/conferencesacrl/ DiscForum2006.htm>, besucht am 2.4.2007) die Standards der Association of College and Research Libraries (ACRL) und Peter Godwin (Keeping up with the Google generation: the challenge for information literacy teachers; in: Information literacy: recognising the need. Ed. Geoff Walton and Alison Pope. Oxford 2006, S. 30-36) die „sieben Säulen“ von SCONUL (Society of College, National and University Libraries), um die Veränderungen in Folge des Web 2.0 in Bezug auf Informationskompetenz zu beschreiben.

25 Lloyd, Annemaree: Information literacy landscapes: an emerging picture. In: Journal of Documentation 62 (2006) S. 570-583. Lloyd hat speziell den Umgang mit Information am Arbeitsplatz z. B. bei Feuerwehrleuten untersucht, wobei sie neben textbezogener Information auch Formen der sozialen und physikalischen Information unterscheidet und damit den Begriff von Informationskompetenz erweitert.

${ }^{26}$ Ingold, Marianne: Informationskompetenz: ein (neues) Leitbild für betriebliche Informationsstellen? In: Leitbild Informationskompetenz: Positionen, Praxis, Perspektiven im europäischen Wissensmarkt; 27. Online-Tagung der DGI, 57. Jahrestagung der DGI, Frankfurt am Main, 23. bis 25. Mai 2005; proceedings. Hrsg. von Marlies Ockenfeld. Frankfurt a. M. 2005. Hier S. 15-26. 


\begin{tabular}{|c|c|c|}
\hline & $\begin{array}{l}\text { Kritische } \\
\text { Informationskompetenz (2.0) }\end{array}$ & $\begin{array}{l}\text { Informationskompetenz } \\
\text { (klassisch) }\end{array}$ \\
\hline $\begin{array}{l}\text { Selbstverständnis und Betonung } \\
\text { liegt auf }\end{array}$ & $\begin{array}{l}\text { Verständnis für das gesamte System wis- } \\
\text { senschaftlicher Information (Produktion, } \\
\text { Verteilung, ...) }\end{array}$ & $\begin{array}{l}\text { Standards und Qualifikationen zum Umgang } \\
\text { mit Information }\end{array}$ \\
\hline $\begin{array}{l}\text { Auffassung von Bildung und Er- } \\
\text { ziehung }\end{array}$ & $\begin{array}{l}\text { Als Prozess, um Reflexionsfähigkeit und } \\
\text { kritisches Bewusstsein zu schaffen }\end{array}$ & $\begin{array}{l}\text { Als Transfer von Information und „richtigem“ } \\
\text { Wissen vom wissenden Lehrenden }\end{array}$ \\
\hline Auffassung von Wissen & $\begin{array}{l}\text { Als Ergebnis eines sozial verhandelten, er- } \\
\text { kenntnistheoretischen Prozesses }\end{array}$ & $\begin{array}{l}\text { Als kulturelles und ökonomisches Kapital, das } \\
\text { unabhängig vom Wissenden existiert }\end{array}$ \\
\hline $\begin{array}{l}\text { Auffassung vom Informations- } \\
\text { prozess }\end{array}$ & Als nicht-linearer, komplexer Prozess & $\begin{array}{l}\text { Als linearer Prozess mit aufeinander folgenden } \\
\text { Schritten }\end{array}$ \\
\hline $\begin{array}{l}\text { Auffassung zur Förderung von } \\
\text { Informationskompetenz }\end{array}$ & $\begin{array}{l}\text { Steigerung von Erkenntnis und Bewusst- } \\
\text { heit (Wie wird Information produziert und } \\
\text { verteilt?) }\end{array}$ & $\begin{array}{l}\text { Lernen von Recherche- und Navigationsstrate- } \\
\text { gien (Wie wird Information gefunden?) }\end{array}$ \\
\hline $\begin{array}{l}\text { Sicht des Nutzenden bzw. Stu- } \\
\text { dierenden }\end{array}$ & Als Mitproduzenten & Als Kunden, als Hilfsbedürftigen \\
\hline Blick auf die Bibliothek & $\begin{array}{l}\text { Als Raum für individuellen oder gemein- } \\
\text { schaftlichen Erfahrungs- und Lernraum }\end{array}$ & $\begin{array}{l}\text { Als Warenhaus für Wissen und Information, als } \\
\text { Informations-Bank }\end{array}$ \\
\hline Rolle des Bibliothekars & $\begin{array}{l}\text { Als Lernberater und Moderator von Infor- } \\
\text { mations- und Lernprozessen }\end{array}$ & Als Lehrender \\
\hline
\end{tabular}

Tabelle 2: Unterscheidung von kritischer und klassischer Informationskompetenz ${ }^{32}$

vorhandener individueller Informationskompetenz. Damit ist Informationskompetenz auch thematisch nah beim individuellen Wissensmanagement ${ }^{27}$. Bibliotheken können durch ihre Serviceangebote auch diesen Bereich berücksichtigen, z. B. durch Beratung zur Nutzung bzw. durch das Angebot von Literaturverwaltungs-Systemen. Insgesamt erscheint mir eine Sicht auf Informationskompetenz bedeutsam, die in einer Reihe von Veröffentlichungen als "critical information literacy" beschrieben wird. Zu nennen sind hier vor allem Autoren wie James Elmborg ${ }^{28}$, Barbara Fister ${ }^{29}$ und Cushla Kapitzke ${ }^{30}$. Tabelle 2 stellt diese kritische der klassischen Sicht auf Informationskompetenz gegenüber. Diese kritische Informationskompetenz betont Aspekte, die zum Tragen kommen, wenn man darüber reflektiert, wie ein dem Web 2.0 angemessenes Verständnis von Informationskompetenz erreicht werden kann. Relevanter werden aber auch gesellschaftliche Aspekte des Umgangs und der Produktion von Information und Wissen in einer so genannten Informationsgesellschaft, in der der Zugang zu den Informationsquellen und das geistige Eigentum von wachsender gesellschaftlicher Bedeutung sind. Diese Gesellschaft entwickelt sich aber für Scott Lash auch in Richtung einer "disinformation society" angesichts der wachsenden Flut und Vielfalt an Informationsmöglichkeiten sowie oft kritiklosem Konsumentenverhaltens ${ }^{31}$.

\subsection{These 2: Informationskompetenz 2.0 ist eine von vielen Schlüsselkompetenzen}

Informationskompetenz 2.0 ist im universitären Umfeld immer Teil einer Vielzahl weiterer, oft verwandter Schlüsselkompetenzen im Bereich wissenschaftlichen Arbeitens und effektiver Studientechniken sowie berufsbezogener Anforderungen. Informationskompetenz wird auch als Meta-Kompetenz bezeichnet ${ }^{33}$, die das Erlernen neuer Fähigkeiten und neuen Wissens erst ermöglicht. Sie umfasst also nicht nur Informationssuche und kann nicht isoliert vom Lernen gesehen werden. Für Mandy Lupton ist gar der Wert von allgemeinen, isolierten Kursen zur Informationskompetenz zweifelhaft: „The value of generic, standalone, parallel and foundation courses for IL [information literacy] education is dubios" 34 . Trotzdem sollen natürlich diesbezügliche Aktivitäten von Bibliotheken nicht aufgegeben werden, sie dürfen nur nicht überbewertet werden. Andererseits kann die Förderung von Informationskompetenz nicht nur eine Aufgabe der Bibliotheken sein. Diese ist auch Aufgabe der Hochschulen, von deren Absolventen Schlüsselkompetenzen für lebenslang notwendiges Lernen erwartet werden. Je mehr Universitäten erkennen und dies dann auch vermarkten, dass ihre Absolventen bestimmte Kompetenzen haben müssen, um lebenslang lernen und im Arbeitsmarkt bestehen zu können, um so

${ }^{27}$ Reinmann-Rothmeier, Gabi und Heinz Mandl: Individuelles Wissensmanagement: Strategien für den persönlichen Umgang mit Information und Wissen am Arbeitsplatz. Bern 2000.

${ }^{28}$ Elmborg, James: Critical information literacy: implications for instructional practice. In: Journal of Academic Librarianship 32 (2006) S. 192-199. Siehe auch Elmborgs persönliche Homepage: Elmborg Home <http://mingo.info-science. uiowa.edu/ elmborg/>, besucht am 2.4.2007.

${ }^{29}$ Fister, Barbara: Smoke and mirrors: finding order in a chaotic world. In: Research Strategies 20 (2006) S. 99-107. Siehe auch Fisters persönliche Homepage: Barbara Fister <http://homepages.gac.edu/ fister/>, besucht am 2.4.2007.

30 Kapitzke, Cushla: Information literacy: a review and poststructural critique. In: Australian Journal of Language and Literacy 26 (2003) S. 53-66. Siehe auch Kapitzkes Homepage: Staff pofil <http://education.qut.edu.au/ kapitzkc>, besucht am 2.4.2007.

${ }^{31}$ Lash, Scott: Critique of information. London 2002.

32 Inhaltlich teilweise nach Elmborg (Anm. 28).

33 Lloyd, Annemaree: Information literacy: the meta-competency of the knowledge economy? An exploratory paper. In: Journal of Librarianship and Information Science 35 (2003) 2, S. 87-92.

${ }^{34}$ Lupton, Mandy: The learning connection: information literacy and the student experience. Adelaide 2004. Hier S. 89. 
größer ist die Chance von Bibliotheken, in diesem Themenfeld Teil der Hochschulaktivitäten zu sein.

Die These dieses Abschnittes impliziert den Vorschlag, bibliothekarisch gesehene Informationskompetenz nicht als den Nabel der Welt zu betrachten. Auf Informationskompetenz gibt es nicht nur vielfältige Sichten (siehe These 1), zusätzlich ist Informationskompetenz nur eine von vielen neuartigen und verwandten Kompetenzen oder Quasi-Synonymen, die im Rahmen der elektronischen Globalisierung eine Rolle spielen: Medienkompetenz, digitale Kompetenz, „e-literacy“ und vieles weitere mehr. Man kann sich sicher lange streiten, welche Begriffe umfassender sind. Vielleicht vernachlässigt Medienkompetenz eher Fragen des Zugangs zu Information und Medien, während im Themenfeld Informationskompetenz - vor allem im Rahmen von Bibliotheken - oft Bereiche der Nutzung, Evaluation und Produktion von Information unterrepräsentiert sind, die in anderen Definitionen stärkeres Gewicht haben ${ }^{35}$. Ist heute vielleicht visuelle Kompeten ${ }^{36}$ fast wichtiger als Informationskompetenz? Man spricht sogar schon von „multiliteracies“, die notwendig sind, um in der modernen Informationsgesellschaft zu bestehen ${ }^{37}$.

Auf die Problematik der Verknüpfung der Begriffe Information und Kompetenz bzw. im Englischen „literacy“ sowie auch auf die diskussionswürdige Übertragung des englischen „literacy“ ins deutsche „Kompetenz“ kann hier nur verwiesen werden ${ }^{38}$. Das englische „information literacy“ impliziert schon vom Begriff her ein Defizit-Modell des Kunden, das nicht damit zusammenpasst, dass Bibliotheken Aufmerksamkeit und Reflexionsfähigkeit wecken und eine Dienstleistung anbieten wollen.

Auf der Ebene von Universitäten ist das Thema Informationskompetenz eingebettet und in Konkurrenz zum gesamten Bereich der Schlüsselkompetenzen und deren Diskussion im Bereich der Einführung der Bachelor- und Master-Studiengänge im Rahmen des Bologna-Prozesses. Informationskompetenz ist dort nur ein, wenn auch aus meiner Sicht sehr wichtiger Teil des Spektrums an Schlüsselkompetenzen. Der Begriff wird dort allerdings selten explizit verwendet. Ein holistisches Verständnis von Informationskompetenz ist bei den Softs Skills nicht nur Teil der Methodenkompetenz (Lern- und Arbeitstechniken), sondern gehört auch zur Sozialkompetenz (Kommunikations- und Kooperationsformen) und Selbstkompetenz (ethisches, eigenverantwortliches Verhalten) ${ }^{39}$.

An vorderster Front der Entwicklung zur Förderung von Schlüsselkompetenzen stehen Universitäten, die ihr ganzes Curriculum nach diesem Ziel ausrichten. So durchlaufen Studierende an der Universität St. Gallen in der Schweiz am Anfang ein einjähriges wissenschaftliches Propädeutikum, Assessment-Stufe genannt. Auch später werden zusätzlich zum Fachstudium im Rahmen eines so genannten Kontextstudiums Handlungskompetenz, Reflexionskompetenz und Kulturelle Kompetenz gefördert ${ }^{40}$. Implizit ist Informationskompetenz hier Teil der Handlungskompetenz (im Bereich der Methoden wissenschaftlichen Arbeitens und Lernens). Zur Reflexions- und kulturellen Kompetenz gehören aber auch Fragen zur Kultur und Natur wissenschaftlichen Arbeitens, ja zu Fragen der Wissenschaftstheorie, -geschichte und -soziologie ${ }^{41}$. Kulturelle Kompetenz umfasst nicht nur das Kennenlernen fremder (Landes-)Kulturen und Sprachen, sondern auch ein vernetztes, interdisziplinär orientiertes Verstehen der
Kulturen fremder Fächer. So hat Informationskompetenz sicherlich auch eine große Bedeutung für so etwas wie „interdisziplinäre Kompetenz"42. Studierende begreifen sich so als Teil einer fachlichen Diskussions- und Diskurs-Gemeinschaft mit eigenen kulturellen und sozialen Strukturen, die ein gemeinsames Vokabular und eine typische Informationspraxis teilt. Eine solche von Birger Hjoerland im Bereich der Informationswissenschaft so bezeichne-

${ }^{35}$ Einen guten Überblick über die Vielfalt dieser Kompetenzen bietet das Buch Digital literacies for learning. Ed. by Allan Martin and Dan Madigan. London 2006.

${ }^{36}$ Marcum, James W.: Beyond Visual Culture: The Challenge of Visual Ecology. In: portal: libraries and the academy 2 (2002) 2, S. 189-206.

${ }^{37}$ Multiliteracies: literacy learning and the design of social futures; [New London Meeting in September 1994]. Ed. by Bill Cope ... for the New London Group. London 2000.

38 Siehe den Aufsatz von Pawley (Anm. 7). Nicht trivial ist auch die Frage nach der wechselseitigen Definition der Begriffe Information und Wissen, siehe dazu z. B. Hapke, Thomas: Zur Diskussion um den Informationsbegriff - eine Rezension (zu Sascha Ott: Information: zur Genese und Anwendung eines Begriffs. Konstanz 2004, auch in: Auskunft 25 [2005] S. 266-271). Preprint: <http://doku.b.tu-harburg.de/ volltexte/2006/248/>, besucht am 2.4.2007.

39 Zur Unterscheidung der Schlüsselkompetenzen siehe das Positionspapier: Schlüsselkompetenzen in den Curricula der Hochschulen / ZEvA Zentrale Evaluations- und Akkreditierungsagentur Hannover. Online: <http://www. zeva.uni-hannover.de/eiqa/Standards SK.pdf>, besucht am 2.4.2007. Mehr zum Thema Kompetenzen siehe z. B. Erpenbeck, John und Lutz von Rosenstiel: Einführung, S. IX-XL. In: Handbuch Kompetenzmessung: erkennen, verstehen und bewerten von Kompetenzen in der betrieblichen, pädagogischen und psychologischen Praxis. Stuttgart 2003, und Schaeper, Hildegard und Kolja Briedis: Kompetenzen von Hochschulabsolventinnen und Hochschulabsolventen, berufliche Anforderungen und Folgerungen für die Hochschulreform. Kurzinformation HIS A6. Hannover 2004.

40 Siehe Spoun, Sascha: Die neukonzipierte Lehre der Universität St. Gallen und ihr Kontextstudium als ein Weg zu nachhaltiger Bildung. In: Nachhaltige Bildung: Hochschule und Wissenschaft im Zeitalter der Ökonomisierung. Peter Hünermann, ... (Hg.). Bielefeld 2005, S. 107-125. Online in: Spoun, Sascha: Bologna Reform: Chance auf Kompetenzentwicklung? In: Förderung von Schlüsselkompetenzen an Hochschulen - Integration von Wissenschaft und Employability? / SKIBA-Projekt (Schlüsselkompetenzen im Bachelor) der Universität Hannover. Dokumentation des Expertenworkshops vom 2. November 2005, S. 25-48. Online: <http://www.wa.uni-hannover.de/wa/skiba/2006-05-03SKIBA-EWS.pdf>, besucht am 2.4.2007.

${ }^{41}$ Siehe zur Wissenschaftssoziologie z. B. Weingart, Peter: Wissenschaftssoziologie. Bielefeld 2003, der auch Fragen zur Wissenschaftskommunikation, zur ,sozialen Konstruktion' des Wissens und Wissensproduktion behandelt, alles Themen, die zu den thematischen Dimensionen von Informationskompetenz gehören können.

42 Siehe dazu Defila, Rico und Antonietta Di Giulio: Vorbereitung auf interdisziplinäres Arbeiten - Anspruch, Erfahrungen, Konsequenzen. In: Neues Handbuch Hochschullehre. Ergänzungslieferung Nummer 9, September 2003. Berlin 2002-2005. E 1.3., S. 1-26. 
te „sozio-kognitive“ Sicht ${ }^{43}$ ist ganz nah bei einem holistischen Verständnis von Informationskompetenz und betont die Bedeutung der historischen Entwicklung dieser Gemeinschaften und ihrer Kommunikationsprozesse sowie die Bedeutung ihrer Strukturen, Dokumenttypen und Institutionen im Rahmen wissenschaftlicher Kommunikation und Information.

So sollten z. B. gerade in den Natur- und Ingenieurwissenschaften Veranstaltungen zur Wissenschaftstheorie sowie Wissenschafts- und Technikgeschichte verpflichtender Teil eines jeden Studiums sein. Hier können unter anderem Arbeits- und Studientechniken bzw. Techniken oder Methoden wissenschaftlichen Arbeitens (Informations- und Literatursuche, Lesemethoden, Exzerpieren, wissenschaftliches Schreiben und Zitieren sowie Präsentation) reflektiert und praktisch geübt werden. Aber auch eine Veranstaltung „Wandel der Wissenschaftskommunikation" mit den Themen Publikation, Wissenschaftssystem, Zitation und wissenschaftliche Reputation, Digitalisierung, Open Access und Urheberrecht, wie sie einmal an der Universität Göttingen stattfand, wäre ein wichtiges Lehrangebot. Im Idealfall könnten dann solche Veranstaltungen von Bibliotheken zusammen mit den Lehrenden genutzt werden, um Elemente der Informationskompetenz und die kulturelle Bedeutung der Überlieferung von Wissen und damit auch die Funktion von Bibliotheken bewusst zu machen ${ }^{44}$.

\subsection{These 3: Informationskompetenz 2.0 ist eine „Lernerfahrung “"45}

Informationskompetenz ist nur kontext- und fachspezifisch als Teil der Erfahrungen, die beim Lernen gemacht werden, vermittelbar. Nur die Lehrenden können die Informationskompetenz ihrer Studierenden wirklich beeinflussen. Entscheidend sind nicht Extra-Kurse zur Vermittlung von Informationskompetenz, sondern der "subtile“ Zwang, sich in projekt-ähnlichen Studienteilen Informationskompetenz erarbeiten zu müssen. Zeit und Ort der Vermittlung von Informationskompetenz müssen so in das Curriculum integriert werden, dass die Studierenden aus ihrer Eigenverantwortung und aus ihrer Studienaufgabe heraus die Kompetenz des Umganges mit elektronischer Fachinformation selbst gesteuert erlernen. Der Zusammenhang zwischen einem Problem und dem Einsatz von Informationskompetenz als Teil der Lösung schafft Motivation, sich diese anzueignen.

Aus einer ganzheitlichen, konstruktivistischen Sicht ist ideales Lernen aktiv, selbst geleitet, situativ und kontextspezifisch, umfasst individuellen Hintergrund und eigene Interpretation. Als sozialer Prozess schafft es Gemeinschaften. Lernziele entstehen oft erst während des Lernprozesses. Problem- und projektorientiertes Lernen im Rahmen der Neuorientierung der Lehre und des Lernens an den Universitäten umfasst vielfältige Kontexte und Perspektiven und ermöglicht eigene forschende Entwicklung. Es ist dabei zu helfen, seinen eigenen Lern- und damit auch Informationsstil zu entwickeln. Relevant sind Beratung (Consulting) zur selbst gesteuerten Optimierung der Informationskompetenz, verbunden mit der Anregung zur Reflexion über den eigenen Lern- und Informationsprozess und dessen Fortschritte. Jedes Lehren sollte Lernen ermöglichen und damit Raum schaffen für die Lernenden, so dass diese diejenigen neuen Einsichten, Fähigkeiten und Potentiale entwickeln können, die von ihnen erwartet werden ${ }^{46}$. Studierende und Lernende sollten nicht als Kunden, sondern als Mitproduzenten angesehen werden, wie es der aus St. Gallen kommende Präsident der Universität Lüneburg in einem Vortrag ausdrückte ${ }^{47}$.

Besonders wichtig ist aber das Lernen und der Austausch der Studierenden untereinander, z. B. im Rahmen eines Projektes. Dieses Lernen entspricht am ehesten dem Lernen in Alltag und Beruf, das eher einem Lernen gleicht, das durch moderne Computerterminologie als „Peer-topeer (P2P)" beschrieben werden kann. Wie bei modernen Netzgemeinschaften, die Software, Musik und Ähnliches austauschen, stellt jeder Teilnehmende der Gemeinschaft einen Teil seiner eigenen Ressourcen (Wissen, Fertigkeiten) im Netzwerk zur Verfügung und partizipiert für seine eigenen (Lern-)Ziele von den Angeboten der Partner der Gemeinschaft. Das „E“ im E-Learning - und damit wird auch die Nutzung sozialer Software im Web 2.0 thematisiert, die man auch als E-Learning bezeichnen kann, - bedeutet nicht nur „electronic“, sondern kann auch als „easy“, „effective“ oder „entertaining“ gelesen werden. In allgemeinster Weise kann es mit „enhanced“ verbunden werden ${ }^{48}$. „Erweitertes“ Lernen umfasst heutzutage mehr als formelle Lernszenarien in Schule, Ausbildung oder Universität. Informelles Lernen, „over-the-shoulder-learning “49 und die Kooperation in Gruppen werden immer wichtiger. Gerade Elemente sozialer Software können zum aktiven, gemeinschaftsorientierten Lernen beitragen, so dass letztlich Lernen wirklich Teil des Lebens wird.

Eine Verknüpfung von sowohl gemeinschaftlichem als auch individuellem Lernen bieten persönliche Lernumgebungen, z. B. in Form von Weblogs, Wikis und elektronischen Portfolios. Zurzeit an den Universitäten genutzte Lernmanagementsysteme sind stark orientiert an den angebotenen Lehrveranstaltungen und wenig orientiert am Lernenden (siehe Tabelle 3). Die massiv wachsende Nutzung der Komponenten des Web 2.0 führt aber immer mehr dazu, dass diese Komponenten auch Teil gängiger Lernplattformen werden. So bietet z. B. die Lernplattform der TU Hamburg-Harburg Stud.IP veranstal-

${ }^{43}$ Vgl. Hjoerland, Birger: Domain analysis. A socio-cognitive orientation for information science research. In: Bulletin of the American Society for Information Science and Technology 30 (2004) 3. S. 17-21.

${ }_{44}$ Vergleiche Hapke, Thomas: Studierende, Google, die Welt der Bibliotheken und deren kulturelle Bedeutung: Ein Essay zu einem Aufsatz von Amy Bruckman. In: Libreas, Ausgabe 5, online: <http://www.ib.hu-berlin.de/ libreas/libreas_neu/ ausgabe5/009hap.htm>, besucht am 2.4.2007.

${ }^{45}$ Nach Lupton (Anm. 34).

${ }^{46}$ Nach Limburg und Sundin (Anm. 20).

47 Siehe auch Anm. 40.

${ }^{48}$ Hapke, Thomas: Perspektive E-Learning - Die Rolle von Universitätsbibliotheken in neuen Lernumgebungen. In: Teaching Library - eine Kernaufgabe für Bibliothekare. Frankfurt a. M. 2007. Hier S. 41-80. Folien des Vortrages unter <http://doku.b.tu-harburg.de/volltexte/2006/310/>, besucht am 2.4.2007.

49 Twidale, Michael B. und Karen Ruhleder: Over-the-shoulder-learning in a distance education environment. In: Learning, culture, and community in online education: research and practice. Eds. Caroline Haythornthwaite und Michelle M. Kazmer. New York 2004. Hier S. 177-194. 
tungsbezogene Wikis an. Sinnvoll wäre also eine Ergänzung der vorhandenen Lernmanagementsysteme durch eine auf den Lernenden bezogene Lernumgebung, wie sie z. B. das System Elgg ${ }^{50}$ bieten würde. Hier haben Lernende die Möglichkeit, eigene Weblogs zu betreiben, ihre RSS-Feeds und Links mit anderen Lernenden zu teilen, aber auch ein Wiki zu nutzen. Darüber hinaus kann die Plattform auch als persönliches elektronisches Portfolio genutzt werden.

\begin{tabular}{|l|l|l|}
\hline & Lernplattformen & $\begin{array}{l}\text { Persönliche Lernumge- } \\
\text { bungen }\end{array}$ \\
\hline $\begin{array}{l}\text { Orientie- } \\
\text { rung an }\end{array}$ & Lehrveranstaltungen & Lernenden \\
\hline Inhalte & $\begin{array}{l}\text { überwiegend Lern- } \\
\text { und Lehrmaterialien } \\
\text { der Lehrenden }\end{array}$ & $\begin{array}{l}\text { überwiegend Lernmate- } \\
\text { rialien der Lernenden }\end{array}$ \\
\hline $\begin{array}{l}\text { Kommu- } \\
\text { nikation }\end{array}$ & $\begin{array}{l}\text { überwiegend zwi- } \\
\text { schen Lernenden } \\
\text { und Lehrenden }\end{array}$ & $\begin{array}{l}\text { überwiegend zwischen } \\
\text { Lernenden }\end{array}$ \\
\hline Beispiele & $\begin{array}{l}\text { Stud.IP, Ilias, } \\
\text { WebCT/Blackboard, } \\
\text { Clixx }\end{array}$ & $\begin{array}{l}\text { Weblogs, Wikis, Elgg, } \\
\text { elektronische Portfolios }\end{array}$ \\
\hline
\end{tabular}

Tabelle 3: Inhalts- und personenbezogene Lernumgebungen

Persönliche Lernumgebungen in Form elektronischer Portfolios $^{51}$ erlauben es $z$. B. jedem Lernenden - aber auch jedem Lehrenden - für sich oder für die eigene Lehrveranstaltung einen Weblog oder ein Wiki zu erstellen und zu nutzen. E-Portfolios bieten Nutzenden die Möglichkeit, die Ergebnisse ihres eigenen Lernens und Lehrens sich und anderen zu präsentieren. Die Nutzenden haben jederzeit die Möglichkeit zu bestimmen, welche Teile des jeweiligen Portfolios nur für einen selbst bzw. für „Bekannte" oder für alle einsehbar sind.

Gerade elektronische Portfolios stellen ein gutes Instrument dar, um nachhaltige und qualitativ hochwertige, auf Reflexion beruhende Lern- und Informationsprozesse zu fördern. Sie können durchaus auch dazu dienen, Anforderungen der Bologna-Studienreform zu berücksichtigen ${ }^{52}$. Der enge Zusammenhang zwischen ihnen und dem Thema Informationskompetenz zeigt folgendes Zitat: „In the context of a knowledge society, where being information literate is critical, the portfolio can provide an opportunity to demonstrate one's ability to collect, organise, interpret and reflect on documents and sources of information. It is also a tool for continuing professional development, encouraging individuals to take responsibility for and demonstrate the results of their own learning. Furthermore, a portfolio can serve as a tool for knowledge management, and is used as such by some institutions." 53

Der konstruktivistisch orientierte Ansatz des Arbeitens mit Weblogs und Wikis kommt modernen Auffassungen vom Lernen entgegen. Rolf Schulmeister führt das Aufkommen der Weblogs auch „auf die häufig recht persönlich motivierte narrative Komponente" zurück ${ }^{54}$. Die das Erzählerische betonende, personenbezogene Komponente eines Weblogs fördert Erinnerung und Reflexion und korreliert mit Empfehlungen aus der wissenschaftlichen Schreibforschung zur Führung von Lern-Tagebüchern und Forschung-Logbüchern ${ }^{55}$. Aber auch ganz allgemein ist das Storytelling schon als Instrument des Lernens in der Hochschulausbildung empfohlen worden ${ }^{56}$. Im Weblog oder elektronischen Portfolio als Instrument reflektierenden Lernens erzählen Schreibende die Geschichte ihres eigenen Lernens und Suchens bzw. Verarbeitens von Informationen, nehmen Gedanken, Ideen und Inhalte auf und sammeln Materialien, Ausarbeitungen und anderes. Sie dienen quasi als eine Dokumentation der eigenen Lernentwicklung, die primär für sich selbst erfolgt, die aber durchaus auch nach außen gegeben werden kann. Narrative Methoden haben in der Form des Story-Telling sogar schon Eingang in das moderne Management gefunden ${ }^{57}$. Im Bereich der qualitativen Forschungsmethoden der Sozialwissenschaften finden sie sich unter dem schönen Begriff „Autoethnography“, also Selbst-Beschreibung der eigenen (Lern-)Kultur ${ }^{58}$. Selbst im Bereich der Ausbildung in den Ingenieurwissenschaften scheint das Thema E-Portfolios ein wichtiger Diskussions- und Forschungsgegenstand zu sein, wie aktuelle Aufsätze auf

50 „Elgg: the open source social networking platform" <http:// www.elgg.org>, besucht am 2.4.2007, und „EduSpaces“ <http://eduspaces.net>, besucht am 2.4.2007.

51 Mehr zu E-Portfolios siehe z. B. unter "E-Portfolios" <http://www.e-teaching.org/didaktik/gestaltung/kommunikation/portfolio/index_html>, besucht am 2.4.2007 sowie „erwachsenenbildung.at - Neue Lernformen - Links“ <http://erwachsenenbildung.at/fachthemen/lernwerkstatt/ links.php?keyword=Portfolio>, besucht am 2.4.2007 oder auf der Homepage von Helen Barrett unter „Dr. Helen Barrett's Electronic Portfolios“ <http://electronicportfolios.com/>, besucht am 2.4.2007.

52 Reinmann, Gabi: Bologna und Web 2.0: Wie zusammenbringen, was nicht zusammenpasst? Vortrag auf der Konferenz eUniversity - Update Bologna am 8. / 9. November 2006 in Bonn. Online: <http://medienpaedagogik.phil. uni-augsburg.de/denkarium/wp-content/uploads/2006/11/ Vortrag_Bonn_Nov06.pdf>, besucht am 2.4.2007.

53 „Why do we need an ePortfolio?" ElfEL (European Institute for E-Learning): <http://www.eife-I.org/publications/ eportfolio/>, besucht am 2.4.2007. Siehe auch den Beitrag von Purdue, J.: Stories, Not Information: Transforming Information Literacy. In: Portal: Libraries and the Academy 3 (2003) 4, S. 653-662.

${ }^{54}$ Schulmeister, Rolf: eLearning: Einsichten und Aussichten. München 2006. Hier S. 275.

55 Spoun, Sascha und Dominik Battiste Domnik: Erfolgreich studieren: ein Handbuch für Wirtschafts- und Sozialwissenschaftler. München 2004. Hier S. 20-22.

${ }^{56}$ McDrury, Janice und Maxine Alterio: Learning through storytelling in higher education: using reflection \& experience to improve learning. London 2003. Vgl. auch: 7 things you should know about digital storytelling. EDUCAUSE Learning Initiative (2007). Online: <http://www.educause.edu/ir/ library/pdf/ELI7021.pdf>, besucht am 2.4.2007.

57 Thier, Karin: Storytelling: eine narrative Managementmethode. Heidelberg 2006. Siehe auch als theoretische Fundierung: Geiger, Daniel: Wissen und Narration: der Kern des Wissensmanagements. Berlin 2006.

58 Holman Jones, Stacy: Autoethonography: making the personal political. In: The sage handbook of qualitative research. Ed. Norman K. Dervin and Yvonna S. Lincoln. 3. ed. Thousand Oaks 2005. Hier S. 763-791. 
den Konferenzen der American Society for Engineering Education zeigen ${ }^{59}$.

Neben der Funktion als Entwicklungs-Portfolio können persönliche Lernumgebungen als so genannte Präsentations-Portfolios auch als Bewertungsinstrument im Rahmen von Lehrveranstaltungen dienen. So ist es für einen Kurs in Informationskompetenz statt einer Klausur oder mündlichen Prüfung sicher sinnvoller, einen bewertbaren Recherche-Portfolio zu verlangen. Andererseits können die öffentlichen Teile von E-Portfolios auch den Lernenden durch sein gesamtes Studium begleiten und später als Alumni lebenslang zur Unterstützung von eigenen Bewerbungen im späteren Berufsleben im Sinne eines Career Service fungieren. Portfolios stellen dann digitale (Kompetenz-)Profile dar, im Sinne persönlicher Webpräsenzen bzw. Vorzeige- oder Bewerbungsportfolios. Es kann durchaus sinnvoll sein, im Intranet einer Universität eine Plattform zum Anlegen von Weblogs, Wikis oder E-Portfolios anzubieten. Ein Angebot von E-Portfolios auf einer Plattform von Universitäten wirkt sicher seriöser und prestigeträchtiger als eine Nutzung von portfolio-ähnlichen Angeboten wie Weblogs und Ähnlichem auf Plattformen wie bloglines.com, wordpress.com u.a. Es gibt zudem auch auf E-Portfolios speziell zugeschnittene Software ${ }^{60}$.

Die Nutzung von Portfolios bzw. deren erzählerische Komponente findet sich wieder im konkreten Suchprozess nach Informationen, der beeinflusst ist vom benutzten Informationssystem, vom individuellen Hintergrund, Wissen und Fähigkeiten des Nutzers sowie vom thematischen, lokalen und gesellschaftlichen Kontext der konkreten Suchanfrage. Hier sind nicht „der" Kontext, "der“ Nutzer oder „das" System wichtig und real, sondern die reale Nutzung eines bestimmten Systems durch einen spezifischen Nutzer zu einem bestimmten Zeitpunkt in einem gerade relevanten Kontext. Nicht die Nutzung als solche ist hier entscheidend, sondern das gerade geschehene Nutzen als einer Art "Mikromoment"61 des Suchens. Nur innerhalb dieses Mikromoments kann in Abhängigkeit von Informationssystem, Nutzer und Kontext wirklich ein Bedürfnis nach Unterstützung und Hilfe beim Recherche- und Informationsprozess notwendig werden. Bis zu diesem Mikromoment hat nun jeder Nutzer schon eine Reise bzw. seine eigene Geschichte („Story“) hinter sich, die genau zu diesem spezifischen Punkt der Nutzung führt. So kann ein Recherche-Portfolio oder ein Informations- oder Lern-Tagebuch genau diese Geschichte widerspiegeln und reflektierbar machen. Informationskompetenz kann wie die Forschung selbst als Teil selbst erzeugter Pfade der Entdeckung gesehen werden ${ }^{62}$. So nutzt ein sehr gelungenes „Lehrbuch“ zum wissenschaftlichen Arbeiten aus den Vereinigten Staaten für Studierende, die ein Studium beginnen, die Metapher des Reisens für das Vertrautwerden mit der wissenschaftlichen Arbeitsweise. Ein Verständnis von Wissenschaft als Diskurs im Laufe der Zeit wird ausgehend vom eigenen Hintergrund und dessen Bedeutung für das eigene Lernen dann erreicht, wenn Studierende selbst zur Forschung beitragen, ihr Wissen mit ihrer Studiengemeinschaft teilen und über ihre Lern- und Informationsprozesse reflektieren ${ }^{63}$.

\subsection{These 4: Informationskompetenz 2.0 umfasst mehr als Lernen mit Information, es ist verstärkt Lernen über Information}

Gerade das Web 2.0 verändert die Inhalte von Informationskompetenz selbst bzw. hebt besonders bisher ver- nachlässigte Dimensionen von Informationskompetenz hervor. Informationskompetenz wird dann nicht nur verstanden als Methodenkompetenz zur besseren Nutzung der Vielfalt der Informationswelt im Sinne der Kenntnis von Recherche- und Navigationsstrategien, sondern auch als Reflexionskompetenz z. B. zu Fragen des geistigen Eigentums oder zu Problemen von Datenschutz und Privatsphäre („privacy“) in der Welt des Web 2.0 und sozialer Software ${ }^{64}$.

Gerade Fragen des geistigen Eigentums wachsen in einer „Cut-and-paste“-Welt. Die Bedeutung und Form des Zitierens von Informationsquellen als Problem der Informationsethik (Plagiarismus) werden immer wichtiger. Aber auch ein Bewusstsein über informationspolitische Aspekte (Urheberrecht, Zugang, Open Access, Datenschutz) ist Teil von Informationskompetenz. Im Rahmen des Studiums muss ernsthaft über Information und ihre Zuverlässigkeit und Seriosität reflektiert werden ${ }^{65}$. Ein Bewusstsein über die soziale Konstruktion von Wissen und Wissenschaft ist genauso notwendig wie eine gezielte Unterstützung zur Vorbereitung künftiger wissenschaftlicher Publikationstätigkeit.

Beispielsweise wird im bis März 2007 abgeschlossenen E-Learning-Projekt der Universitätsbibliothek der TU Hamburg-Harburg mit dem Titel VISION (VIrtual Services for Information ONline) ${ }^{66}$ ein Online-Tutorial zum wissenschaftlichen Arbeiten geschaffen, das - in Ergänzung zum vorhandenen, mehr den Bereich der Recher-

${ }^{59}$ Recherche nach Portfolio unter „ASEE - Conferences Conference Proceedings Serach" <http://www.asee.org/ conferences/v2search.cfm>, besucht am 2.4.2007, z. B. Guan, Z.; Lappenbusch, S. u. a.: Portfolios in engineering education: What do they promise and how can they be used? 2006 ASEE Conference (Überblicksartikel) <http://www. asee.org/acPapers/code/getPaper.cfm?paperID=11601>, besucht am 2.4.2007. Ein Beispiel aus der Verfahrenstechnik ist Broadway, F.S.; Qammar, H.K.; Evans, E.A. und S. Spickard-Prettyman: The use of reflective journals for student learning and development. In: Frontiers in Education, 2005. FIE ,05. Proceedings 35th Annual Conference 19-22 Oct. 2005 Page(s): F2C - 13-19. Online unter Digital Object Identifier: <http://dx.doi.org/10.1109/FIE.2005.1612042>, besucht am 2.4.2007 bzw. <http://ieeexplore.ieee.org/iel5 /10731/33854/01612042.pdf?tp=\&arnumber=1612042\&is number $=33854>$, besucht am 2.4.2007.

60 Siehe auch: Electronic portfolio. (2007, March 21). In: Wikipedia, The Free Encyclopedia, <http://en.wikipedia.org/ w/index.php?title=Electronic_portfolio\&oldid=116829971>, besucht am 2.4.2007.

61 Bruce, Harry: The user's view of the Internet. Lanham, Md. 2002, S. 60.

62 Siehe auch Purdue (Anm. 53).

${ }^{63}$ Watts, Margit Misangyi: College: we make the road by walking. 2. ed. Upper Saddle River, NJ 2006.

${ }^{64}$ Siehe auch Abschnitt 2.2 oben, aber auch Barnes, Susan: A privacy paradox: social networking in the United States. First Monday 11 (2006) 9. Online: <http://firstmonday.org/ issues/issue11_9/barnes/>, besucht am 2.4.2007.

65 Siehe den Aufsatz von Bruckman, Amy S.: Student research and the internet. In: Communications of the ACM 48 (2005) 12, S. 35-37. Dazu auch Hapke (Anm. 44).

66 Siehe <http:// www.vision.tu-harburg.de>, besucht am 2.4.2007. 
che nach Informationen abdeckenden Tutorial DISCUS ${ }^{67}$ - vor allem die Problematik der Produktion von Information wie Lesen, Schreiben und Publizieren, verbunden mit Aspekten wie Zeitschriftenkrise, Open Access, Geistiges Eigentum und Urheberrecht thematisiert. Das Tutorial demonstriert, dass Visualisierung nicht nur Mittel der Gestaltung, sondern auch Mittel zur konstruktiven Wissensvermittlung unter Einbeziehung dramaturgischemotionaler Elemente sein kann ${ }^{68}$. Es stellt kein umfassendes Tutorial zum wissenschaftlichen Arbeiten dar, sondern soll ein Problembewusstsein in diesem Bereich vermitteln, verbunden mit weiterführenden Angeboten, die integriert in Umgebungen des Web 2.0 zusätzliche Informationen bieten, z. B. in einem Wiki, mit Linklisten in „Social-Bookmarking-Diensten“, durch Buchvorschläge über librarything.com u.a.

Ein gelungenes Beispiel zum Thema „Zitieren im Rahmen wissenschaftlichen Arbeitens" wird in einem Aufsatz von Bettina Berendt mit einem Einstieg über Google verknüpft. Die Relevanzkriterien von Google zur Darstellung seiner Suchergebnisse beruhen auch auf dem Zitieren, hier dem Verlinken, von Webseiten. Es geht nicht nur um das "Wie“ des Zitierens, sondern vor allem auch um das „Warum"69! Das Thema Plagiate kann durchaus als eines der wichtigsten Punkte einer zukünftigen Informationskompetenz gesehen werden. Angesprochen werden können hier auch einige tiefgehende Problemstellungen, z. B.: Was ist ein Autor? Wie entsteht überhaupt eine neue Idee? Was bedeutet Kreativität hinsichtlich von Ideen? Im Zeitalter von Open Access und Creative Commons sind die Grenzen zwischen Plagiat und originalem Beitrag in einem gewissen Sinne fließend. Eigentlich kann man sich heutzutage nicht sicher sein, dass eine Idee wirklich die eigene ist. Könnte es nicht sein, dass man bei dem Information Overload, der täglich auf einen einwirkt, manches wahrnimmt, vergisst und dieses plötzlich aus dem Unterbewusstsein als „eigene Idee" wieder auftaucht? Ist die Gestaltung eines eigenen Weblogs, der aus gefilterten Beiträgen der Welt der Information besteht, irgendwann eine eigene geistige Leistung? Kreativität kann heute auch heißen, aus der vorhandenen Informationsvielfalt bestimmte Aspekte herauszupicken, diese neu zusammenzustellen und fertig ist ein neues Produkt oder eine neue Idee. Wahrscheinlich gibt es so etwas wie eine alte und eine neue Kreativität bezüglich von Ideen!? Es gibt ja auch verschiedene Formen von Kreativität. Kann der Begriff Schöpfungshöhe aus dem Urheberrecht wirklich definiert werden oder ist er nicht eher ein Produkt einer Aushandlung zwischen gesellschaftlichen Kräften?

Das bestehende Urheberrecht, das ja erst im Laufe des Buchdruckes und gesellschaftlicher Entwicklung entstanden ist, muss kritisch reflektiert werden. Selbst der Begriff des Autors kann ja als gesellschaftliche Konstruktion angesehen werden! Alternative Lizenz-Modelle wie Creative Commons betonen z. B. gerade das „Share, reuse, and remix - legal"70 und die damit mögliche Förderung von Kreativität. Das Entscheidende bei der Plagiatsproblematik im Rahmen wissenschaftlichen Arbeitens ist sicherlich das korrekte Zitieren bzw. generell die Namensnennung, wenn man Ideen und Werke anderer verwendet. Aber was, wenn sich die Welt der Information in Richtung der von Jorge Luis Borges beschriebenen Bibliothek von Babel entwickelt, die alle Bücher oder elektronischen Werke enthält, die aus der Kombination von allen möglichen Zeichen kombinatorisch konstruiert werden können. Dies ist dann eine Welt, in der kein Plagiat möglich ist bzw. alle Werke Plagiate sind. Sind wir auf dem Weg dorthin?
Der Umgang mit dem Überfluss an Informationen, verbunden mit Unterstützung zu dessen Bewältigung, gehört ebenfalls zum Lernen über Information ${ }^{71}$.Das im vorigen Abschnitt erwähnte Storytelling gehört zusammen mit den Werkzeugen des Web 2.0 zu den wichtigen Möglichkeiten, um dem Information Overload zu begegnen ${ }^{72}$. Aber auch die Problematik des Umgangs mit sozialer Software und dem Web 2.0 erweitert die inhaltlichen Dimensionen von Informationskompetenz erheblich. Nach Lambert Heller umfasst Informationskompetenz auch die Fähigkeit, die sozialen Netzwerke des Web 2.0 so zu benutzen, um eigene Ideen in Netzwerken zu verbreiten und weiterzuentwickeln ${ }^{73}$. Schon lange gibt es Konzepte, die die Teilnahme an praxisbezogenen Gemeinschaften von Personen, die informell miteinander verbunden sind und ähnlichen Aufgaben gegenüber stehen („,community of practice“) $)^{74}$, als Lernen bezeichnen ${ }^{75}$.

${ }^{67}$ Siehe DISCUS <http://discus.tu-harburg.de>, besucht am 2.4.2007, und Bieler, Detlev; Hapke, Thomas und Oliver Marahrens: Lernen, Informationskompetenz und Visualisierung - Das Online-Tutorial DISCUS (Developing Information Skills \& Competence for University Students) der Universitätsbibliothek der TU Hamburg-Harburg. In: ABI-Technik 25 (2005) 3, S. $162-181$.

${ }^{68} \mathrm{Vgl}$. Detlev Bieler unter <http://www.vision.tu-harburg.de/ info>, besucht am 2.4.2007.

69 Berendt, Bettina: Studentische Literaturarbeit in Zeiten des World Wide Web. In: Berendt, B.; Voss, H.-P. und J. Wildt (Eds.): Neues Handbuch Hochschullehre (G3.3, pp. 1-30). Berlin 2003. Online: <http://warhol.wiwi.hu-berlin.de/ berendt/ Papers/berendt 2003.pdf>, besucht am 2.4.2007. Überarbeitete und aktualisierte Version als Berendt, Bettina: Studentische Literaturarbeit in Zeiten des World Wide Web. In: Berendt, B.; Voss, H.-P. und J. Wildt (Eds.): Neues Handbuch Hochschullehre - Best Of. Berlin 2006. Siehe auch Weber, Stefan: Das Google-Copy-Paste-Syndrom: wie Netzplagiate Ausbildung und Wissen gefährden. Hannover 2007.

70 Creative Commons <http://creativecommons.org/>, besucht am 2.4.2007.

${ }^{71}$ Hilfreich sind hier Arbeiten von Martin J. Eppler, z. B.: Eppler, Martin J. und Jeanne Mengis: The concept of information overload: a review of literature from organization science, accounting, marketung, MIS and related disciplines. In: The Information Society 20 (2004) S. 325-344. Eppler berücksichtigt auch Visualisierungen und hat die verschiedenen Visualisierungsmethoden selbst in einem Periodensystem visualisiert: Visual Literacy: An E-Learning Tutorial on Visualization for Communication, Engineering and Business $<$ http://www.visual-literacy.org/pages/documents.htm>, besucht am 2.4.2007.

72 Sax, Boria: Storytelling and the „information overload“. In: On the Horizon 14 (2006) 4, S. 165-170.

${ }^{73} \mathrm{Vgl}$. den Beitrag „Informationskompetenz 2.0: Drei aktuelle Überlegungen zum Lernen durch Weblogs" im Weblog netbib vom 19.1.2007 unter <http://log.netbib.de/?p=78607016>, besucht am 2.4.2007.

${ }^{74}$ Artikel Community of Practice. In: Wikipedia, Die freie Enzyklopädie. Bearbeitungsstand: 19. März 2007, 08:00 UTC. <http://de.wikipedia.org/w/index.php?title=Community_of_ Practice\&oldid=29350462>, besucht am 2.4.2007.

75 Graham Attwell nennt als Beispiel das Konzept der „legitimen periphären Partizipation" von Lave, Jean und Etienne Wenger: Situated learning: legitimate peripheral participation. Cambridge 1991. Zitiert in Attwell, Graham: E-Learning und die soziale Gestaltung der Technik. In: Bittlinger, Uwe H. und Ulrich Bauer (Hrsg.): Die „Wissensgesellschaft“: Mythos, Ideologie oder Realität? Wiesbaden 2006. Hier S. 523-550. 
Zusammenfassend gilt, dass es heutzutage nicht nur wichtig ist, mit und durch Information zu lernen, sondern Reflexion und Lernen über Information und Wissen anzuregen bzw. zu ermöglichen. Ein solcher kritischer Ansatz erweitert das Konzept von Informationskompetenz in Richtung einer "hyperliteracy“, die neben kritischer Kompetenz beim Umgang mit Texten unter anderem auch den kritischen Umgang mit Bildern berücksichtigt ${ }^{76}$. Das, was als Informationskompetenz oder auch als "net savvy" bezeichnet werden kann, ändert sich mit der Weiterentwicklung der $\mathrm{Netze}^{77}$.

\subsection{These 5: Informationskompetenz 2.0 steht für die Nutzung von Komponenten sozialer Soft- ware durch Bibliotheken als Dienstleistende für das Lernen ihrer Kunden}

Eine große Bandbreite von Aktivitäten zur Informationskompetenz-Förderung und im Auskunfts-Service ist nötig, um die Kunden in ihrem aufnahmefähigen („teachable“) Moment $^{78}$ zu erreichen. Nur durch ein weites Spektrum werden diverse Zielgruppen und Lerntypen erreicht. Dieses umfasst z. B. integrierte oder auch außerhalb von Lehrveranstaltungen stattfindende Präsentationen oder gar eigene Lehrveranstaltungen der Bibliothek, Online-Tutorials, ,just-in-time“-Beratung bei der realen oder virtuellen Auskunft im Chat, Newsletter per Email, Plakate, Lesezeichen, Broschüren usw. Darüber hinaus müssen Bibliotheken in Zukunft daran arbeiten, die Komponenten sozialer Software für eigene Dienstleistungen zu nutzen bzw. den Kunden an den Orten zu begegnen, an denen sie sich befinden. Diese Orte sind heute Teil des Web 2.0! Bibliotheken sollten daher Weblogs, Wikis, Podcasts aber auch andere Komponenten des Web 2.0 wie My Space, Second Life usw. nutzen, um ihre Kunden zu erreichen ${ }^{79}$. Weblogs können als Neuigkeiten-Dienst über Serviceangebote der Bibliothek dienen und dabei die Kommunikation mit den Kunden (Kommentarfunktion) erhöhen. Die Staats- und Universitätsbibliothek Hamburg nutzt dieses Instrument erfolgreich seit dem Jahre $2006^{80}$. Wikis dienen als bequemes Hilfsmittel, um gemeinsam mit Nutzern Seiten zur Fachinformation anzubieten ${ }^{81}$. Podcasts vermitteln in möglichst erzählerischer, persönlicher Form Neuigkeiten und Wissenswertes zu Bibliothek und Information, wie es die Versuche der Universitätsbibliothek der TU Hamburg-Harburg zeigen ${ }^{82}$. Die Übersicht über Weblogs und andere Informationsangebote behält man - wenn vorhanden - mit RSS-Feeds ${ }^{83}$. Genauso wie Bibliotheken ihren Kunden Carrels als Lese- und Arbeitskabinen zur Verfügung stellen, um dort wissenschaftliche Arbeiten, z. B. Dissertationen, ungestört bearbeiten zu können, könnten sie persönliche Lernumgebungen in Form von Weblogs, Wikis und E-Portfolios als eine Form von elektronischen Carrels in ihren Hochschulen anbieten.

Das im Rahmen eines BMBF-Projektes entwickelte Assistenzsystem BibTutor ${ }^{84}$ ist zwar kein Produkt des Web 2.0, ist aber ein Serviceangebot, bei dem der Nutzer am „Point of need“ Beratung abfordert. Hier wird eine Form von „Just-in-time-E-Learning“85 geboten. Direkte Links aus der konkreten Recherchesituation heraus führen über BibTutor unmittelbar zu entsprechenden Seiten mit weiterführenden Informationen als Informationsmodule von BibTutor oder als externe Lernmodule wie z. B. Online-Tutorials wie DISCUS oder LOTSE ${ }^{86}$. Mehrere kleine Lern-
Sequenzen entsprechen den Nutzerbedürfnissen meist besser als ein umfangreiches Tutorial. Die Bedeutung des Kontextes und der "Mikromomente" des Recherchierens werden durch BibTutor, aber auch durch die Werkzeuge des Web 2.0 gefördert!

\section{$3 \quad$ Bibliotheken im Web 2.0}

Der von Bibliotheken zurzeit gern verwendete Begriff Teaching Library ${ }^{87}$ deckt nur einen Teilbereich des möglichen Dienstleistungsspektrums von Bibliotheken bei

${ }^{76}$ Kapitzke, Cushla: Information literacy: a positivist epistemology and a politics of outformation. In: Educational Theory 53 (2003) 1, S. 37-53.

77 Siehe auch die Net Savvy Series mit den Beiträgen „Ensuring the Net Generation Is Net Savvy“, "Getting Past Google: Perspectives on Information Literacy from the Millennial Mind“ und „How Choice, Co-Creation, and Culture Are Changing What It Means to Be Net Savvy" aus dem Jahre 2006 auf der Website der Educause Learning Initiative unter „ELI Resources" <http://www.educause.edu/ELIResources/10220>, besucht am 2.4.2007.

78 Block, Marylaine (2003): Teach them while they're asking for information: reference as a teachable moment. In: Net effects: how librarians can manage the unintended consequences of the Internet. Medford, NJ 2003, S. 76-79.

79 Siehe z. B. Deitering und Bridgewater (Anm. 24) sowie Godwin, Peter: Information literacy in the age of amateurs: how Google and Web 2.0 affect librarians' support of Information Literacy. In: ITALICS 5 (2006) 4. Online: <http://www. ics.heacademy.ac.uk/italics/vol5iss4/godwin.pdf>, besucht am 2.4.2007.

80 „Neues aus Stabi und Fachbibliotheken“ < http://www.sub. uni-hamburg.de/blog/>, besucht am 2.4.2007.

81 Vgl. Schröter, Marcus: Fünf Jahre nach SteFi oder: Auf der Suche nach Informationskompetenz im Studienalltag. Von der ,Ware' Information zur ,wahren' Information - Erstellen einer Fachinformationsseite Geschichte von Studierenden für Studierende. In: Bibliotheksdienst 40 (2006) S. 1 2861295.

82 „TUBCast - unser 2. Podcast ist online!“ <http://www.tub. tu-harburg.de/4438.html>, besucht am 2.4.2007.

${ }^{83}$ Hier ein Beispiel für eine Feedsammlung zu Zeitschriften der Verfahrenstechnik, zu Dokumentenservern sowie zu Weblogs von Bibliotheken und zur wissenschaftlichen Kommunikation: <http://www.bloglines.com/public/thapke/>, besucht am 2.4.2007. Ein Versuch, Kunden die Mächtigkeit der Hilfsmittel des Web 2.0 zu demonstrieren, stellt die sehr schnell zusammenstellbare Homepage des Autors beim Dienst Pageflakes dar: <http://www.pageflakes.com/t-hapke.ashx>, besucht am 2.4.2007.

${ }^{84}$ Vergleiche Anm. 6.

${ }^{85}$ Back, Andrea; Bendel, Oliver und Daniel Stoller-Schai: ELearning im Unternehmen: Grundlagen - Strategien - Methoden - Technologien. Zürich 2001. Hier S. $193 \mathrm{ff}$.

86 Siehe „Willkommen bei LOTSE!" <http://lotse.uni-muenster. de>, besucht am 2.4.2007.

87 Siehe auch den Titel des sonst sehr lesenwerten Buches von Lux, Claudia und Wilfried Sühl-Strohmenger: Teaching library in Deutschland: Vermittlung von Informations- und Medienkompetenz als Kernaufgabe für Öffentliche und Wissenschaftliche Bibliotheken. Wiesbaden 2004. 


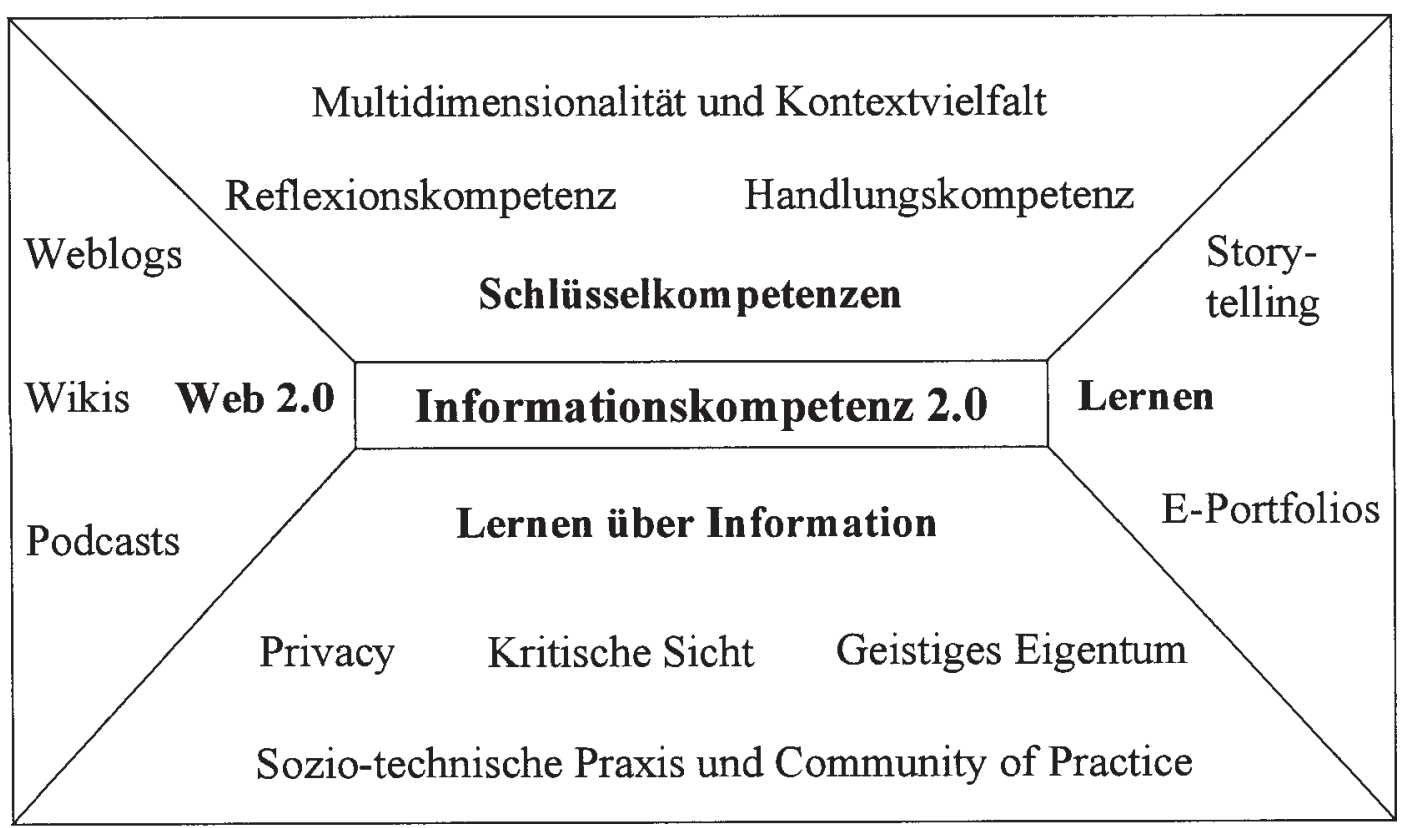

Abb. 1: Informationskompetenz 2.0

der Unterstützung des Lernens ab. Wenn möglich und sinnvoll sollen und müssen in Bibliotheken Arbeitende auch Lehrveranstaltungen und Schulungen durchführen. Aber das Lernen zu ermöglichen und zu unterstützen, ist die wichtigste Rolle für Bibliotheken im Universitätsbereich. Besser ist daher der Begriff „Learning Facilitating Library", da er neben der inhaltlichen Dimension der Informationskompetenz auch eine räumliche (Bibliothek als physischer Lernort) und organisatorische Dimension (z. B. Zusammenarbeit mit anderen Serviceeinheiten, Realisierung eines Single-Sign-On u.a.) umfasst, die Bibliotheken berücksichtigen müssen ${ }^{88}$. Auch Dienstleistungen von Bibliotheken im Bereich E-Learning, das selbst immer mehr von Komponenten des Web 2.0 beeinflusst wird, gehören hier dazu. Diese können z. B. die Zusammenstellung „elektronischer Semesterapparate", die neben Link-Tipps auch extra für die betreffende Lehrveranstaltung digitalisierte Dokumente als elektronische Lernobjekte enthalten können (mit Abklärung der Urheberrechte), sowie die Verknüpfung von Bibliotheksdienstleistungen mit Lernplattformen umfassen.

Trotz Tendenzen zu Monopolisierung und Standardisierung wird die Komplexität und Vielfalt der Welt der Information und der Datenbankangebote für die Kunden von Bibliotheken immer größer. Unsicherheit über die Qualität von Rechercheergebnissen in elektronischen Informationssystemen wird trotzdem nicht von jedem wahrgenommen. Google und zusammenfassende Portalangebote unterstützen die Orientierung, ohne jemals aufgrund der vorhandenen Diversität alle Angebote des so genannten „Deep Web“ erfassen zu können. Bibliotheken bieten ein Abbild der Mannigfaltigkeit und Verschiedenheit der Welt der Information. Sie fördern mit ihren Aktivitäten im Bereich Informationskompetenz Reflexion und Bewusstheit beim Umgang mit dem Informationsdschungel, ohne die eine „informationelle Au- tonomie"89 lebenslang Lernender und mündiger Bürger nicht möglich ist.

Eine das Lernen fördernde Bibliothek bietet ihren Kunden neben dem physischen Lernort auch Beratungsangebote und die Möglichkeit für die Kunden, ihr Repertoire an Erfahrungen beim Suchen und Finden von Informationen auszuweiten und zu verändern. Bibliotheken müssen versuchen, auf das Verschwinden des Nutzers zu reagieren und diesen als direkt Beteiligten in ihre Informationsangebote einbinden. Mehr noch, sie müssen in den Umgebungen potentieller Kunden mit ihren Dienstleistungen und Ressourcen sichtbar bleiben im Sinne des von der American Library Associations Office for Information Technology Policy beauftragten Reports mit dem Titel „Participatory Networks" 90 . Dabei betont besonders der Untertitel „The Library as Conversation“ die hoffentlich stärker werdende teilnehmende Rolle von Bibliotheken am wissenschaftlichen und öffentlichen Diskurs. In der Welt des Web 2.0 werden nicht nur die Nutzer mehr als bisher am „Bestand“ der Bibliothek teilhaben und deren Wissensbasis mit aufbauen, sondern auch in Bibliotheken Arbeitende öffnen sich verstärkt und pro-aktiv ihrer jeweiligen Umwelt.

${ }^{88}$ Hapke, Thomas: ,In-formation' of Better Learning Environments - the Educational Role of the University Library. In: LIBER Quarterly 15, 3/4, S. 178-199. Preprint: <http://eprints. rclis.org/archive/00006592/>, besucht am 2.4.2007.

${ }^{89}$ Siehe Kuhlen (Anm. 4).

90 Lankes, R. David; Silverstein, Joanne und Scott Nicholson: Participatory networks: the library as conversation. American Library Associations Office for Information Technology Policy, 2007. Online: <http://iis.syr.edu/projects/PNOpen/>, besucht am 2.4.2007. 
Vielleicht ist eine beratende Rolle besser als eine lehrende Rolle bei Informationskompetenz-Aktivitäten der Bibliotheken? Gerade im Bereich der Problematik des geistigen Eigentums, der auch in Deutschland wichtig werdenden Problematik des Plagiarismus oder im Bereich der Bewertung von Forschungspublikationen mit Zitat-Datenbanken ist im Alltag immer wieder die beratende Rolle der Bibliotheken gefragt ${ }^{91}$. Dabei wäre vielleicht eine systemisch orientierte Lernberatung ein möglicher Weg, eine Beratung, die neben der Autonomie der zu beratenden Person immer auch die Autonomie der sozialen Systeme und Kontexte - hier der Fachwissenschaft bzw. der Studierenden-Situation in der spezifischen Organisation Universität - berücksichtigt ${ }^{92}$. Informationskompetenz verändert sich mit der sich ändernden Informations-(Um)welt. Auf jeden Fall gilt: Die Bibliothek 2.0 fördert Informationskompetenz 2.0 (vgl. Abb. 1). Die Zukunft gehört einer Bibliothek, „where librarians become more critical commentators, mediators and mentors - perhaps nomadic intellectuals and cultural tourists - rather than traditional archivists and monitors"93.

\section{Anschrift des Autors:}

Thomas Hapke

Technische Universität Hamburg-Harburg

Universitätsbibliothek

D-21071 Hamburg

E-Mail: hapke@tu-harburg.de

91 Ein aktuelles Werk, das eine ganzheitliche Sicht auf Bibliotheken und deren Serviceangebote und dabei auch Fragen des Ortes bzw. des Raumes Bibliothek sowie Probleme der Wissensproduktion berücksichtigt, ist: Libr@ries: changing information space and practice. Ed. Cushla Kapitzke und Bertram C. Bruce. Mahwah, NJ 2006.

92 König, Eckard und Gerda Volmer: Systemisch denken und handeln: personale Systemtheorie in Erwachsenenbildung und Organisationsberatung. Weinheim 2005.

${ }^{93}$ Luke, Allan und Cushla Kapitzke: Literacies and libraries: archives and cybraries. In: Pedagogy, culture and society 7 (1999) S. 467-491. Hier S. 476. 